\title{
Determinación de la diversidad genética de la paloma doméstica Columba livia (Columbidae) a partir de genes polimórficos asociados con el color del plumaje en San Antero, Córdoba, Colombia
}

\author{
(ㄱ) Adrián Enrique Rodríguez-De La Barrera ${ }^{1, *}$, ๑Luis Alfonso Causil-Vargas ${ }^{1}$, Orlando Causil-Vargas ${ }^{1,2}$ \\ ${ }^{1}$ Departamento de Biología, Facultad de Ciencias Básicas, Universidad de Córdoba, Montería, Colombia \\ ${ }^{2}$ Departamento de Ciencias Naturales y Educación Ambiental, Facultad de Educación y Ciencias Humanas, \\ Universidad de Córdoba, Montería, Colombia
}

\begin{abstract}
Resumen
El objetivo de esta investigación fue determinar la diversidad genética de la población de la paloma doméstica Columba livia empleando genes polimórficos asociados con el color del plumaje en San Antero, Colombia. Entre marzo y abril del 2017 se hicieron muestreos aleatorios en cuatro subpoblaciones del municipio de San Antero ubicadas en los sitios de Calle Abajo, Calle Central, Parque Central e Iglesia Central mediante excursiones urbanas, observación directa y registros fotográficos, y se clasificaron fenotípicamente 235 palomas. Se estudiaron los marcadores autosómicos Grizzle (G); Spread (S); Checker (C) y Ash-Red (B). Los perfiles genéticos de las subpoblaciones de palomas domésticas se establecieron con los siguientes índices genético-poblacionales: las frecuencias alélicas, la diversidad genética según Nei (1972), la heterocigocidad esperada (He), el coeficiente de diferenciación genética $\left(\mathrm{G}_{\mathrm{st}}\right)$, el flujo génico $(\mathrm{Nm})$ y las distancias genéticas entre las poblaciones utilizando el programa PopGene 1.31. Los índices de fijación propuestos por Wright, $\mathrm{F}_{\mathrm{is}}, \mathrm{F}_{\mathrm{it}}$ y $\mathrm{F}_{\mathrm{st}}$, se calcularon mediante el programa FSTAT v 2.9.3.2. Los marcadores más frecuentes fueron el Checker y el Spread, en tanto que el Ash-Red evidenció las menores frecuencias alélicas. En la población total hubo un alto porcentaje de homocigotos y la diferenciación genética fue baja. Se sugieren posibles efectos de selección en los marcadores Checker y Spread. (C) 2019. Acad. Colomb. Cienc. Ex. Fis. Nat.
\end{abstract}

Palabras claves: Estructura genética; Frecuencia alélica; Flujo genético; Heterocigocidad.

Determination of the genetic diversity of the domestic pigeon Columba livia (Columbidae) employing polymorphic genes associated with the color of the plumage in San Antero, Córdoba, Colombia

\begin{abstract}
The objective of the study was to determine the genetic diversity of the domestic pigeon Columba livia using polymorphic genes associated with the plumage color in San Antero, Colombia. Between March and April 2017, random samplings were carried out in four sites of the municipality of San Antero located in Calle Abajo, Calle Central, Parque Central, and Iglesia Central. During urban excursions and through direct observation and photographic records, we made the phenotypic classification of 235 pigeons. The markers studied were Grizzle (G), Spread (S), Checker (C), and Ash-Red (B). The genetic profiles of the populations of domestic pigeons were established with the following indices: allelic frequencies, Nei's genetic diversity (1972), expected heterozygosity $(\mathrm{He})$, genetic differentiation coefficient $\left(\mathrm{G}_{\mathrm{st}}\right)$, gene flow $(\mathrm{Nm})$, and genetic distances between populations were estimated using the PopGene 1.31 software. We calculated the fixation indices proposed by Wright: $\mathrm{F}_{\mathrm{is}}, \mathrm{F}_{\mathrm{it}}$ and $\mathrm{F}_{\mathrm{st}}$ using the FSTAT v 2.9.3.2 program. The most frequent markers were Checker and Spread while Ash-Red showed the lowest values. The total population showed a high percentage of homozygotes and genetic differentiation was low. Checker and Spread markers suggested possible selection effects. (C) 2019. Acad. Colomb. Cienc. Ex. Fis. Nat.
\end{abstract}

Key words: Genetic structure; Allelic frequency; Gene flow; Heterozygosity.

\section{Introducción}

La paloma doméstica Columba livia (Gmelin, 1789) pertenece a la familia Columbidae, la cual probablemente evolucionó mediante procesos de selección a partir de especies de aves antecesoras. A menudo denominada paloma bravía de Castilla o zuro, se la considera una especie invasora (González, et al., 2018) que se asienta en hogares y se mantiene como un ave de ornato (Mancera, et al., 2013); tiene un elevado crecimiento poblacional debido a su capacidad adaptativa en los entornos de gran incidencia humana

\footnotetext{
*Correspondencia:

Adrián Enrique Rodríguez-De La Barrera; adrian.norssate@gmail.com

Recibido: 20 de noviembre de 2018

Aceptado: 11 de marzo de 2019

Editor: Alberto Gómez
} 
(Mondocorre, et al., 2014), lo que le permite desarrollarse bajo diversas condiciones urbanas (Vargas, 2016) y, probablemente, responde a cierto tipo de selección artificial (Jacquin, et al., 2011).

El plumaje de la paloma doméstica presenta variaciones (Lesiss, et al., 1999a), y las bases genéticas de la coloración y el plumaje se codifican mediante un sistema múltiple de alelos (Dunmore, 1968). Asimismo, existen 60 factores hereditarios que influyen en la coloración de la paloma doméstica (Gibson, 1995). Los genes que codifican la pigmentación de la melanina tienen efectos pleiotrópicos sobre otros procesos biológicos de las aves (Gasparini, et al., 2009). Los procesos adaptativos bajo los cuales se fija la coloración y la melanización resultan de factores de la historia natural de las aves, por lo que se cree que las palomas de diferentes colores están adaptadas a ambientes alternativos cuya abundancia de alimentos (Roulin, 2004) y la exposición a los parásitos varían (Miranda, 2006).

Los marcadores fenotípicos asociados con la coloración son herramientas útiles para analizar los perfiles genéticos de las poblaciones naturales; la información obtenida permite inferir las relaciones genealógicas entre los individuos de una población, la valoración del flujo genético entre poblaciones, estimar el tamaño efectivo de las poblaciones y minimizar los efectos negativos de la endogamia (Eiroa, 2016).

Varios autores han reportado estudios genético-poblacionales de palomas domésticas en Colombia. En Bogotá se ha hecho la caracterización genética de la población de palomas domésticas (Pardo, et al., 2015): los marcadores Checker y Spread fueron los de mayor frecuencia alélica $\mathrm{y}$, asimismo, se evidenció un elevado flujo génico y bajas distancias genéticas. En Lorica, Córdoba, se evaluó la diversidad genética de las palomas utilizando genes asociados con el color (Causil, et al., 2016), registrándose la escasa diferenciación genética entre las poblaciones y la posible selección natural para el marcador Spread. La diversidad genética implica aspectos importantes en términos de la adaptación y el mejoramiento de las especies (Díaz, et al.,
2004). En ese contexto, el propósito de este estudio fue analizar la diversidad genética de la paloma doméstica $(C$. livia) a partir de los genes asociados con el color del plumaje en San Antero, Córdoba.

\section{Materiales y métodos}

Área de estudio. El estudio se llevó a cabo en San Antero, municipio ubicado en la costa norte de Colombia a $9^{\circ} 22^{\prime}$ $28,711^{\prime \prime} \mathrm{N}$ y $75^{\circ} 45^{\prime} 30,661$ " O, cuya temperatura promedio es de $28^{\circ} \mathrm{C}$. Se muestrearon cuatro subpoblaciones ubicadas en los sitios de Calle Abajo, Calle Central, Parque Central e Iglesia Central.

Recolección de los datos. Entre marzo y abril del 2017, se hicieron muestreos aleatorios en cuatro subpoblaciones del municipio durante excursiones urbanas, mediante la observación directa y con ayuda de registros fotográficos en horas de la mañana (de 6:00 am a 9:00 am). Se hizo una clasificación fenotípica de cada uno de los individuos adultos encontrados $(\mathrm{n}=235)$, atendiendo a la presencia o ausencia de los marcadores autosómicos (Tabla 1) Grizzle $(G)$, Spread (S), Checker (C) y Ash-Red (B).

Diseño estadístico. Los perfiles genéticos de las poblaciones se calcularon con los siguientes índices genéticopoblacionales: las frecuencias alélicas de cada marcador a nivel poblacional y global; las medidas de diversidad genética establecidas por Nei (1973) correspondientes a la heterocigocidad esperada $(\mathrm{He})$, la heterocigocidad esperada de la población total (HT), el coeficiente de diferenciación genética $\left(\mathrm{G}_{\mathrm{st}}\right)$, el flujo génico $(\mathrm{Nm})$, y las distancias genéticas de Nei (1972), de las poblaciones, las cuales se estimaron utilizando el programa PopGene 1.31 (Yeh, et al., 1999). La estructura genética de las poblaciones según los índices de fijación propuestos por Wright $\left(\mathrm{F}_{\mathrm{is}}, \mathrm{F}_{\mathrm{it}} \mathrm{y} \mathrm{F}_{\mathrm{st}}\right)$, se calculó mediante el programa FSTAT v 2.9.3.2 (Goudet, 2002).

\section{Resultados}

Se muestrearon 235 individuos distribuidos en cuatro subpoblaciones (Figura 1): Calle Abajo $(n=42)$, Calle Central $(n=63)$, Parque Central $(n=83)$ e Iglesia Central $(n=47)$.

Tabla 1. Descripción de los cuatro genes estudiados

\begin{tabular}{l|cl}
\hline Locus & Alelos & Característica \\
\hline Grizzle (gen autosómico) & G & Mutante, produce machas blancas extendidas alrededor de la cabeza o el cuerpo de la paloma. \\
\hline Spread (gen autosómico) & g & Silvestre, sin machas blancas \\
\hline Checker (gen autosómico) & C & Mutante, origina palomas negras. \\
& s & Silvestre, palomas que no son negras \\
& c & Sutante, produce un patrón de manchas triangulares gris claro o azul sobre fondo oscuro en el \\
Ash-Red (gen ligado al sexo) & B & Mutante, produce palomas rojas. \\
& b & Silvestre, origina palomas que no son rojas. \\
\hline
\end{tabular}

Grizzle (G); Spread (S); Checker (C) y Ash-Red (B) 

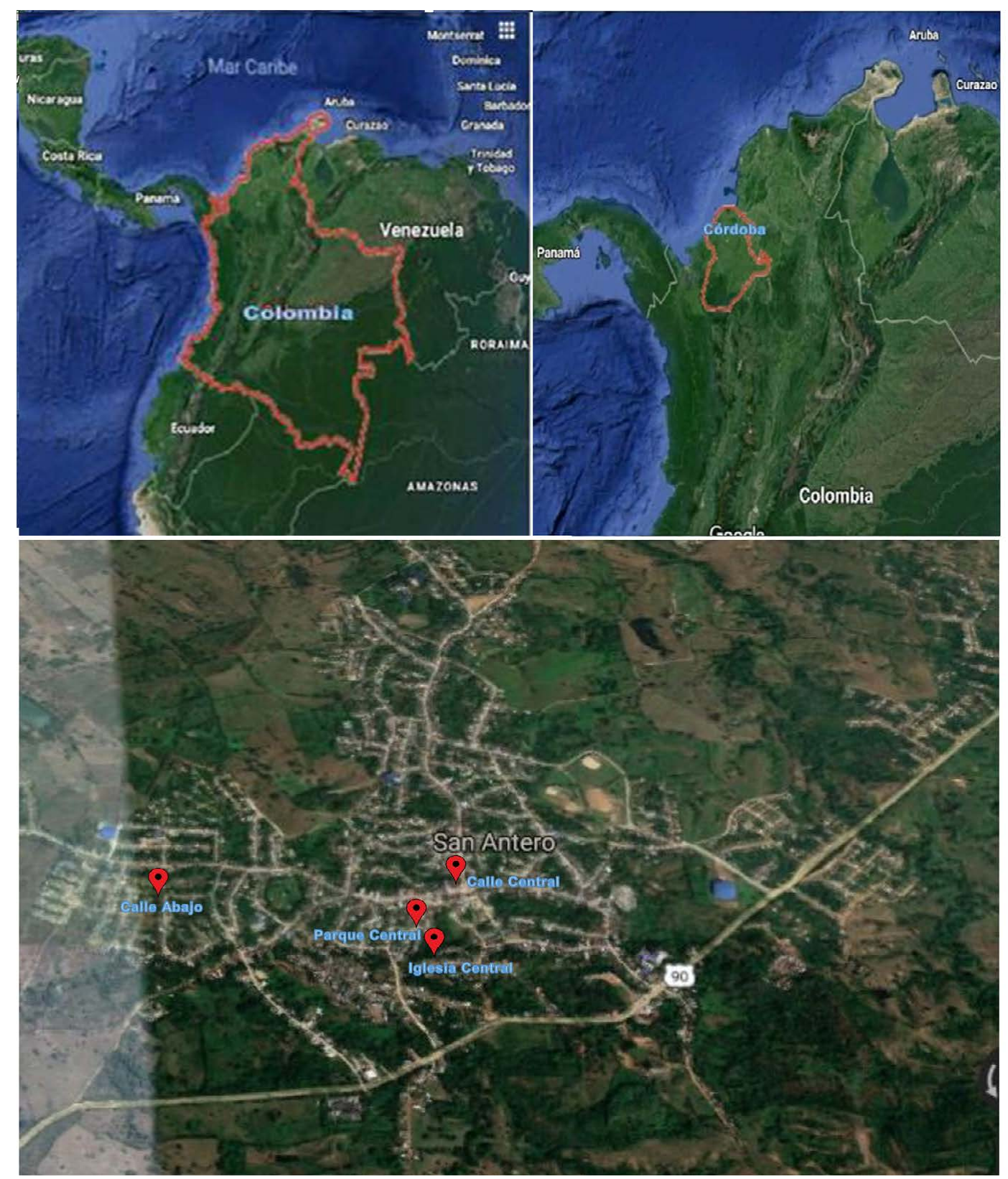

Figura 1. Subpoblaciones de palomas domésticas muestreadas en San Antero-Córdoba, Colombia.

Frecuencias alélicas. Las frecuencias alélicas para cada población (Tabla 2) evidenciaron que el marcador Checker $(\mathrm{p}=0,4293)$ fue el más frecuente, seguido de Spread ( $\mathrm{p}=0,3683)$ y de Ash-Red, con los valores más bajos $(\mathrm{p}=0,0729)$.

Diversidad genética. La mayor heterocigocidad esperada se encontró en el marcador Spread, con un valor de 0,4005, y la más baja, en el marcador Ash-Red, con un valor de 0,1158 (Tabla 3). Los valores de la heterocigocidad observada fueron bajos: oscilaron entre un máximo de 0,1667 para el marcador Checker y un mínimo de 0,0278 para el Ash-Red. En cada marcador los valores de la variabilidad genética fueron relativamente bajos.
Coeficiente de diferenciación genética. El nivel de diferenciación genética (Tabla 4) entre las distintas subpoblaciones de palomas domésticas (C. livia) en la población de San Antero fue relativamente bajo según el valor promedio de $\mathrm{F}_{\text {st }}$, el cual fue de 0,0634 , con un +inidce de heterogeneidad entre poblaciones $\left(\mathrm{G}_{\mathrm{st}}\right)$ igual a 0,037 , lo cual indica que aproximadamente el $3,7 \%$ de la variación revelada se debe a la diferencia entre las poblaciones y, por lo tanto, las cuatro subpoblaciones no fueron significativamente diferentes en cuanto a los marcadores estudiados.

El valor del flujo génico $(\mathrm{Nm}=2.870)$ evidenció que las poblaciones mantenían el intercambio genético, asumiéndose un total aproximado de tres migrantes por generación. 
Tabla 2. Frecuencias alélicas de cada marcador en las poblaciones estudiadas

\begin{tabular}{lcccc}
\hline \multirow{2}{*}{ Poblaciones } & \multicolumn{4}{c}{ Locus } \\
\cline { 2 - 5 } & G & S & C & B \\
\hline Calle Abajo & 0,2361 & 0,1111 & 0,6111 & 0,0694 \\
Calle Central & 0,2500 & 0,3750 & 0,2875 & 0,1500 \\
Parque Central & 0,2195 & 0,5545 & 0,4818 & 0,0445 \\
\hline Iglesia Central & 0,2495 & 0,4327 & 0,3370 & 0,0277 \\
Total & 0,2387 & 0,3683 & 0,4293 & 0,0729 \\
\hline
\end{tabular}

G: Grizzle; S: Spread; C: Checker; B: Ash-Red

Tabla 3. Índice de diversidad genética de Nei (1973) en cada marcador a través de la heterocigocidad esperada (He), la heterocigocidad observada (Ho) y el valor de p para cada marcador

\begin{tabular}{lccc}
\hline Locus & He & Ho & p \\
\hline Grizzle & 0,2878 & 0,0278 & 0,1342 \\
Spread & 0,4005 & 0,0556 & 0,1261 \\
Checker & 0,3983 & 0,1667 & 0,1440 \\
Ash-Red & 0,1158 & 0,0278 & 0,1089 \\
Promedio & 0,3006 & 0,0695 & \\
\hline
\end{tabular}

Tabla 4. Coeficiente de diferenciación genética $\left(\mathrm{G}_{\mathrm{ST}}\right)$ y flujo génico $(\mathrm{Nm})$ en San Antero

\begin{tabular}{ccc}
\hline $\mathbf{G}_{\text {ST }}$ & $\mathbf{N m}$ & $\mathbf{p}$ \\
\hline 0,037 & 2,870 & 0,0710 \\
\hline
\end{tabular}

Cabe señalar que el valor obtenido está por encima de 1 , lo que indica que el flujo génico supera los efectos de la deriva génica en las subpoblaciones y previene la diferenciación local. Ello significa que las subpoblaciones de palomas domésticas se comportaron como una sola y sugiere que para un mejor análisis y una comprensión espacio-temporal dinámica, no se debería establecer una subestructura de la población.

Estructura poblacional. Los valores positivos del $\mathrm{F}_{\text {is }}$ para todos los marcadores (Tabla 5) evidenciaron un exceso de homocigotos entre los individuos comparados con cada subpoblación, lo que lleva a admitir la presencia de consanguinidad dentro de las subpoblaciones, con valores que oscilan entre 0,2401 para el marcador Spread y 0,1125 para el marcador Checker. El valor promedio del $\mathrm{F}_{\text {st }}$ fue de 0,0634 , lo que podría indicar una diferenciación genética significativa entre las poblaciones. Por último, el estadístico $\mathrm{F}_{\text {it }}$ evidenció un exceso de homocigotos de los individuos con respecto a la población total.

Distancias genéticas. La distancia genética (Tabla 6) entre las subpoblaciones fue baja, siendo más cercanas las subpoblaciones de Calle Central e Iglesia Central, en tanto que las subpoblaciones de Calle Abajo y Calle Central resultaron ser las de mayor diferencia genética. La subpoblación de Calle Abajo registró el valor más alto de distancia génica en comparación con el resto de las subpoblaciones.

\section{Discusión}

Los elevados valores de las frecuencias alélicas de los marcadores Checker y Spread en este estudio concuerdan con los resultados descritos en otras investigaciones (Causil, et al., 2016; Pardo, et al., 2015). Los genes asociados con los colores melanóticos en palomas domésticas son más frecuentes en los centros urbanos, ya que, al parecer, las gónadas son más activas en épocas de menos lluvias, de manera que pueden reproducirse en mayor número en una temporada del año, en contraposición con otras aves que tienen diferentes patrones del pelaje (Hetmansky, 2008).

La mayor frecuencia de individuos melanóticos podría obedecer a cierta ventaja selectiva que tienen las palomas domésticas de coloraciones oscuras, ya que se ha reportado que son menos susceptibles de ser atacadas por parásitos hemosporidios; asimismo, serían más resistentes a ciertos patógenos (Lei, et al., 2013). Las coloraciones claras de las plumas en palomas domésticas podrían hacerlas fisiológicamente propensas a los parásitos cuando las ciudades son relativamente grandes; se cree que estos individuos más claros tienen una menor capacidad inmunitaria; por el contrario, los individuos más oscuros tienen una gran actividad inmunológica. Esta relación entre la inmunidad y la coloración con melanina podría deberse a efectos pleiotrópicos resultado de los altos niveles de la melanocortina (Jacquin, $\boldsymbol{e t}$ al., 2013). Otra posible explicación se relacionaría con las expresiones fenotípicas oscuras, ya que estas tienen mayor

Tabla 5. Valores de los estadísticos F para cada marcador en la población global

\begin{tabular}{lcccc}
\hline Marcadores & $\mathbf{F}_{\text {IT }}$ & $\mathbf{F}_{\text {ST }}$ & $\mathbf{F}_{\text {IS }}$ & $\mathbf{p}$ \\
\hline Ash-Red & 0,3065 & 0,0020 & 0,1476 & 0,0090 \\
Grizzle & 0,1416 & 0,1611 & 0,1190 & 0,0071 \\
Spread & 0,2221 & 0,0726 & 0,2401 & 0,0051 \\
Checker & 0,0001 & 0,0182 & 0,1125 & 0,0061 \\
Promedio & 0,1675 & 0,0634 & 0,1548 & \\
\hline
\end{tabular}

Tabla 6. Matriz de distancia genética (Nei, 1972) entre las poblaciones

\begin{tabular}{ccccc}
\hline & 1 & 2 & 3 & 4 \\
\hline 1 & ---- & & & \\
2 & 0,1465 & ----- & & \\
3 & 0,0425 & 0,0356 & ----- & \\
4 & 0,1099 & 0,0026 & 0,0200 & ----- \\
\hline
\end{tabular}

1: Calle Abajo; 2: Calle Central.; 3: Parque Central; 4: Iglesia Central 
resistencia al estrés y es posible que estas palomas tengan exposición perinatal a la corticosterona, lo cual depende de la condición materna, de las características del nido y de las provisiones de alimento, aspectos que, al parecer, tienen efectos organizativos y permiten que los individuos oscuros soporten mejor el estrés en los centros urbanos (Corbel, et al., 2016)

Otro factor asociado a la selectividad de colores oscuros en las palomas domésticas podría deberse a la gran producción de huevos por parte de las palomas más oscuras, hecho que sería atribuible a posibles ventajas selectivas de los individuos con mayor masa corporal, lo que ocasiona un incremento en la actividad reproductiva de las palomas y, probablemente, causaría efectos selectivos de las condiciones ambientales en los genes melanóticos del plumaje (Causil, et al., 2016)

El uso del hábitat puede tener un papel importante en el momento de fijar los genes melanóticos, siendo este el caso de los marcadores polimórficos. Específicamente, es posible que las plumas melanóticas tengan una mayor eficacia de desintoxicación por la intensa actividad de muda de estas aves, lo cual permite suponer que las palomas más oscuras podrían habitar zonas más contaminadas que las palomas con patrones más claros, rasgo que representaría una posible fuerza selectiva a favor de los fenotipos melanóticos entre las palomas domésticas en los centros urbanos (Chatelain, et al. 2014). Era de esperarse que las frecuencias alélicas se acentuaran en los marcadores más oscuros como el Checker y el Spread, pues el estudio se llevó a cabo en un municipio urbano, lo que influiría en que los genes melanóticos exhibieran patrones selectivos como la inmunidad a los parásitos, así como la ventaja fisiológica de la acción de la melanocortina y la gran producción de huevos y el consecuente aumento de estos marcadores, hecho coherente con el grado de urbanización de esta localidad.

El grado de flujo genético permite inferir que existe una fuerza que mantiene integrada la población, lo que condicionaría la persistencia de un perfil genético en la población local, de manera que los rasgos de distribución geográficas evolucionaron de forma conjunta (Cárdenas, 2012).

La reducción de heterocigotos obtenida al aplicar los distintos índices de fijación $\left(\mathrm{F}_{\text {is }}\right.$ y $\left.\mathrm{F}_{\mathrm{it}}\right)$ en cada una de las subpoblaciones estudiadas evidenció que se comportan como una población, en tanto que los bajos índices de diferenciación genética revelan una débil estructuración de la diversidad genética (Vergara, 2015), hecho que sería atribuible a que las poblaciones naturales y comunicadas entre sí exhiben una menor diferenciación genética (Matallanas, 2015). El grado de diferenciación genética entre las poblaciones fue bajo $\left(\mathrm{G}_{\mathrm{st}}=0,037\right)$. Un estudio en aves demostró que los machos se aparean con varias generaciones de su descendencia provocando una creciente similitud entre ellas (Acuña, 2016). Las palomas tienden a elegir parejas genéticamente similares, lo que ocasiona altos niveles de endogamia en la población (Jacob, et al., 2016); por otra parte, es posible que exista una correlación entre las distancias genéticas y las geográficas, pues la diferenciación genética aumenta proporcionalmente con las distancias geográficas, lo que podría implicar un número significativo de inmigrantes (Zuleta, et al., 2012). Esto sugiere que los eventos selectivos conllevaron un intercambio genético que superó los efectos de la deriva genética y la distinción local (Causil, et al., 2016).

Las distancias genéticas entre las cuatro subpoblaciones del estudio, especialmente la cercanía geográfica entre la subpoblación de Calle Central e Iglesia Central, se explicarían por el alto flujo genético entre ellas, ya que, al parecer, las palomas domésticas presentan mayor probabilidad de vuelo de una población cercana a otra, lo que genera en los individuos un mayor grado de confianza y probablemente es producto de su adaptación en zonas urbanas (Skandrani, et al., 2016). Esto podría explicar el parecido genético de las subpoblaciones estudiados y permite concluir que la población de palomas domésticas de San Antero tendría un mismo perfil genético-poblacional, por lo que se deberían estudiar como una sola población.

\section{Conclusiones}

Los marcadores más frecuentes fueron el Checker y el Spread, ya que, al parecer, la selección natural está favoreciendo a los individuos más oscuros por su mayor eficacia reproductiva, cierta resistencia a los parásitos y las ventajas inmunológicas producto de efectos pleiotrópicos. La población total mostró un alto porcentaje de homocigotos y a nivel de las subpoblaciones la diferenciación génica fue baja, lo cual evidencia un parentesco genético entre las palomas de los sitios estudiados debido al intercambio de genes entre estas subpoblaciones y refleja la existencia de similitudes genéticas locales.

\section{Agradecimientos}

Los autores agradecen a los estudiantes de la Escuela Normal Superior Santa Teresita de Lorica, por los registros fotográficos que permitieron la realización de este trabajo.

\section{Financiación}

El estudio fue financiado con recursos propios de los autores.

\section{Contribución de los autores}

Adrián Enrique Rodríguez-De La Barrera se encargó del muestreo, redacción y análisis de manuscrito; Luis Alfonso Causil-Vargas se encargó de interpretar y analizar los diferentes métodos estadísticos aplicados en este estudio y Orlando Causil-Vargas se encargó de procesar los datos estadísticos en los softwares y tabular los datos recolectados.

\section{Conflicto de intereses}

Ninguno que declarar.

\section{Referencias}

Acuña-Rodríguez, W. (2016). Determinación de la diversidad y estructura genética de patos criollos (Cairina moschata L. 1758) 
de los departamentos de Lambayeque y San Martín mediante el uso de microsatélites. Universidad Nacional Mayor de San Marco, Lima, Peru. p. 57-59.

Cárdenas, M. O. M. (2012). Dinámica genético poblacional del pingüino magallánico, Spheniscus magellanicus (Forster, 1781) de isla Guafo, sur de Chile y sus relaciones filogeográficas con poblaciones del Atlántico Sur. Universidad Austral de Chile, Valdiavia, Chile. p. 36-38.

Causil-Vargas, L., Rodríguez-De La Barrera, A., Causil-Vargas, O. (2016). Diversidad genética de palomas domésticas (Columba livia) en Lorica, Colombia, utilizando genes que codifican la coloración del plumaje. Revista de Investigaciones Veterinarias del Perú. 27 (3): 448-457.

Chatelain, M., Gasparini, J., Jacquin, L., Frantz, A. (2014). The adaptive function of melanin-based plumage coloration to trace metals. Biology Letters. 10 (3): 20140164.

Corbel, H., Legros, A., Haussy, C., Jacquin, L., Gasparini, J., Karimi, B., Frantz, A. (2016). Stress response varies with plumage colour and local habitat in feral pigeons. Journal of Ornithology. 157 (3): 825-837.

Díaz, R., Alfonsín, P., Abuín, M., Fernández-López, J. (2004). Caracterización de la variabilidad genética de una población de mejora de Juglans regia L. Investigación agraria. Sistemas y recursos forestales. 13 (3): 518-526.

Dunmore, R. (1968). Plumage polymorphism in a feral population of the rock pigeon. American Midland Naturalist. 79 ( 1 ): 1-7

Eiroa, D. (2016). Caracterización genética de la población de paloma rabiche (Columba junoniae) reintroducida en la isla de Gran Canaria. Universidad de la Laguna. Canarias, Instituto Universitario De Enfermedades Tropicales y Salud Pública de Canarias. p. 1-33.

Gasparini, J., Bize, P., Piault, R., Wakamatsu, K., Blount, J. D., Ducrest, A. L., Roulin, A. (2009). Strength and cost of an induced immune response are associated with a heritable melanin-based colour trait in female tawny owls. J. Anim. Ecol. 78: 608-616.

Gibson, L.P. (1995). Genetics of Pigeons Columba livia (Gmelin). Plain City, USA. p. 180-187.

González-Oreja, J. A., Zuria, I., Carbó-Ramírez, P., Charre, G. M. (2018). Using variation partitioning techniques to quantify the effects of invasive alien species on native urban bird assemblages. Biological Invasions. 20 (10): 1-14.

Goudet, J. (2002). FSTAT 2.9. 3: A program to estimate and test gene diversities and fixation indices (updated from Goudet 1995). Lausanne: Switzerland. p. 485-486.

Hetmański, T. \& Jarosiewicz, A. (2008). Plumage polymorphism and breeding parameters of various feral pigeon Columba livia gm. morphs in urban area Gdańsk, north Poland. Pol. J. Ecol. 56 (4): 683-691.

Jacob, G., Prévot, A. C., Baudry, E. (2016). Feral Pigeons (Columba livia) Prefer Genetically Similar Mates despite Inbreeding Depression. PloS one. 11 (9): e0162451.

Jacquin, L., Lenouvel, P., Haussy, C., Ducatez, S., Gasparini, J. (2011). Melanin-based coloration is related to parasite intensity and cellular immune response in an urban free living bird: The feral pigeon Columba livia. Journal of Avian Biology. 42 (1): 11-15.

Jacquin, L., Récapet, C., Prévot-Julliard, A. C., Leboucher, G., Lenouvel, P., Erin, N., ... Gasparini, J. (2013). A potential role for parasites in the maintenance of color polymorphism in urban birds. Oecologia. 173 (3); 1089-1099.
Lei, B., Amar, A., Koeslag, A., Gous, T. A., Tate, G. J. (2013). Differential haemoparasite intensity between black sparrowhawk (Accipiter melanoleucus) morphs suggests an adaptive function for polymorphism. PLoS One. 8: (12 e8160)

Leiss, A. \& Haag-Wackernagel, D. (1999a). Gefiederfärbungen bei der Strassentaube (Columba livia). J. Ornithol. 140: 341-353.

Mancera, V. M. M., Jiménez, L. C. V., Medina, D. A. B., Soler-Tovar, D. (2013). La paloma (Columba livia) en la transmisión de enfermedades de importancia en salud pública. Revista Ciencia Animal. 6: 177-194.

Miranda, L. (2006). Aislamiento e identificación de patógenos entéricos de heces de palomas en la ciudad de la Paz, Bolivia. Bolivia: Universidad Mayor de San Andrés, Facultad de Ciencias Farmacéuticas y Bioquímicas. p. 15-25.

Matallanas-Peñas, B. (2015). Análisis genético de las poblaciones españolas del cangrejo de río Austropotamobius italicus (Faxon, 1914). Disertación doctoral, Universidad Complutense de Madrid.

Mondocorre, W., Pérez, F., Albis, N., Zeballos, M. (2014). Estimación de la abundancia de palomas (Columba livia) y percepción de la ciudadanía, para su control y manejo en la ciudad de Sucre. Universidad Mayor Pontificia de San Frnacisco Xavier de Chusquisaca. p. 1-20.

Nei, M. (1972). Genetic distance between populations. Amer Natur. 106: 283-292.

Nei M (1973) Analysis of gene diversity in subdivided populations. Proc. Nat. Acad. Sci. 70 (12): 3321-3323.

Pardo, E., Cavadia, T., Alvarino, G. (2015). Análisis de la diversidad genética de la paloma domestica (Columba livia) en Bogotá, Colombia, utilizando genes que codifican la coloración y diseño del plumaje. Bistua Revista de la Facultad de Ciencias Básicas, Universidad de Pamplona. 13 (1): 35-45.

Rincón, J., López, A., Echeverri, J. (2013). Estructura y diversidad genética en vacas Holstein de Antioquia usando un polimorfismo del gen bGH. Revista MVZ Córdoba. 18 (1): 3346-3354.

Roulin, A. 2004. The evolution, maintenance and adaptive function of genetic colour polymorphism in birds. Biol. Rev. 79: $1-34$

Skandrani, Z., Prevot, A. C., Baldaccini, N. E., Gasparini, J. (2016). On the interplay between phylogeny and environment on behaviour of two urban bird species, Columba livia and Corvus corone (Aves). Italian Journal of Zoology. 83 (1): 98-102.

Vargas-García, J. L. (2016). Prevalencia de Cryptosporidium sp. en heces de paloma doméstica (Columba livia) de parques y plazas de la ciudad de Huanuco y su efecto en la salud pública. Universidad de Huanuco, Huanuco, Peru. p. 8-9.

Vergara-Pacheco, D.A. (2015). Diversidad genética de Phytelephas aequatorialis Spruce en la provincia de Manabí. Tesis. Pontificia Universidad Catolica del Ecuador, Facultad de Ciencias Exactas y Naturales.

Yeh, F., Yang, R., \& Boyle, T. (1999). Microsoft Windows-based free ware for population genetic analysis (Release 1.31). University of Alberta, Edmonton. p. 1-3.

Zuleta, T. \& María, A. (2012). Genética poblacional de la lagartija de lava endémica (Microlophus bivittatus) de la Isla San Cristóbal e islote Lobos, Galapagos-Ecuador, mediante microsatélites como parte de la línea base para su manejo y conservación. Tesis. Universidad de San Francisco de Quito, Colegio de postgrado, Quito, Ecuador. 